\title{
LLUVIAS TORRENCIALES EN EL MARESME (BARCELONA) EL 12 DE OCTUBRE DE 2016
}

\author{
Gabriela Cuevas $^{(1)}$, Ramón Pascual ${ }^{(2)}$, Alfons Callado ${ }^{(3)}$, Marc Compte $^{(4)}$ \\ (1)AEMET Cataluña, Arquitecte Sert 1, 08005 Barcelona, gcuevast@aemet.es \\ (2) AEMET Cataluña, Arquitecte Sert 1, 08005 Barcelona, rpascualb@aemet.es \\ (3) AEMET Cataluña, Arquitecte Sert 1, 08005 Barcelona, acalladop@aemet.es \\ (4) AEMET Cataluña, Arquitecte Sert 1, 08005 Barcelona, mcompter@aemet.es
}

\section{Introducción}

Durante el miércoles 12 y el jueves 13 de octubre de 2016 tuvo lugar un episodio de precipitaciones intensas y generalizadas en toda la península ibérica. Un frente frío, asociado a una baja fría centrada al noroeste de Portugal, atravesó la Península causando chubascos frecuentes y ocasionalmente fuertes en puntos del suroeste y centro peninsular. Previamente al paso del frente, la confluencia de diferentes flujos de recorrido mediterráneo configuró una línea de inestabilidad que atravesó el litoral catalán durante la tarde del miércoles 12. Esta línea originó tormentas con chubascos muy fuertes, incluso torrenciales y de larga duración en puntos de la comarca del Maresme (Barcelona), que acumularon más de $200 \mathrm{~mm}$ en diversos lugares en menos de 4 horas. La intensa lluvia provocó importantes daños en infraestructuras y la muerte de una persona.

Los episodios altamente convectivos sobre el Mediterráneo occidental suelen ocurrir en verano y a comienzos del otoño. En Cataluña, el otoño es la estación que concentra la mayor ocurrencia de inundaciones. Estos eventos suelen tener corta duración y pueden conducir al desbordamiento repentino de ríos provenientes de los Pirineos y de arroyos/ torrentes costeros. De hecho, el litoral catalán es una de las regiones más afectadas por episodios de lluvias repentinas y, en particular, el Maresme (Fig. 1) es el área de más

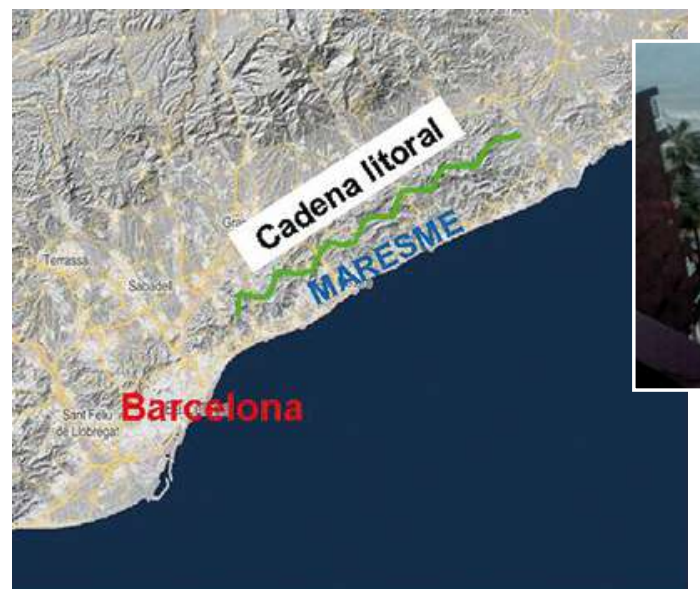

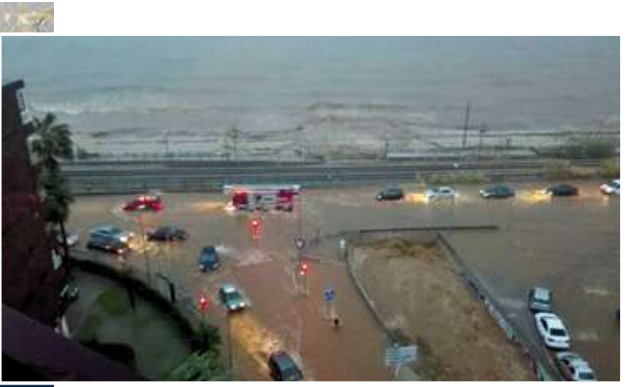

Fig. 1. A la izquierda, situación de la zona donde tuvo lugar el episodio de lluvias torrenciales (Maresme, Cataluña). A la derecha, fotografía que muestra el desbordamiento de rieras y calles anegadas en la confluencia con la N-II en el Maresme. 
impacto así como una de las más pobladas (Pascual, 1999; Chacon et al., 2005). A consecuencia de esto, el riesgo natural asociado a este fenómeno es muy elevado (Llasat et al., 2010).

Este estudio presenta un análisis del entorno sinóptico y mesoescalar del episodio, y de la fenomenología asociada a través de la exploración de imágenes de teledetección, campos de modelos numéricos de predicción y observaciones convencionales. Los impactos del evento también son estudiados a partir del análisis de la información en los medios. Finalmente, se discute la precisión de la predicción mediante el análisis de los avisos de fenómenos meteorológicos adversos emitidos para ese día por AEMET.

\section{Impactos}

El episodio de fuertes precipitaciones hizo que, en al menos 5 ciudades del bajo Maresme, se superasen los $100 \mathrm{~mm}$ acumulados en 24 horas (según registros de 3 redes de estaciones: Aemet, Meteocat y Meteoclimatic; Fig. 2). La lluvia torrencial dio lugar a un récord de intensidad de precipitación en Cataluña, con $85 \mathrm{~mm}$ en media hora registrados en Cabrils.
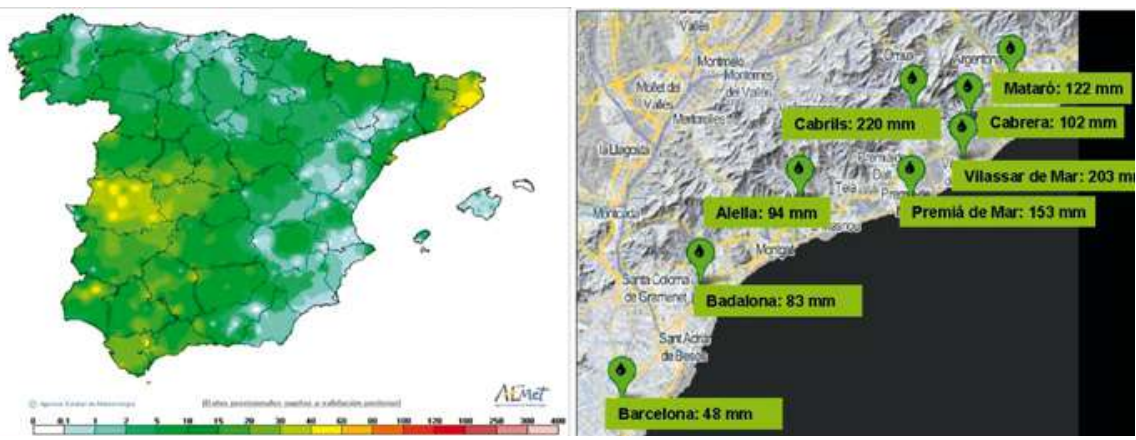

Fig. 2. A la izquierda, precipitación acumulada de 00 UTC del 12 octubre a 00 UTC del 13 octubre en la red de estaciones automáticas de AEMET. A la derecha, registros de precipitación recogidos en diversas redes (AEMET, Meteoclimatic, Meteocat) para la zona del Maresme durante el día 12 de octubre.

Entre los impactos más importantes del evento (Tabla 1) cabe destacar los cortes de tráfico y de la circulación de trenes, cortes de suministro eléctrico, el arrastre por las aguas de coches aparcados junto a rieras (Fig.1) y, desafortunadamente, la muerte de una persona dentro de un coche atrapado en un paso subterráneo.

$\Rightarrow$ Más de 900 llamadas a Emergencias de la Generalitat de Cataluña durante el episodio y 300 al servicio de bomberos. Activada la alerta de inundaciones INUNCAT.

$\Rightarrow$ Carretera nacional N-II y secundaria C-31 cortadas. Servicio de tren suspendido durante varias horas.

$\Rightarrow$ Personas atrapadas en la N-II y en pasos subterráneos inundados tuvieron que ser rescatadas.

$\Rightarrow$ Récord de precipitación en 24 h en Cataluña desde 1943.

$\Rightarrow$ Un hombre muere atrapado en su coche arrastrado por la lluvia en Cabrils. 
$\Rightarrow$ Varias rieras desbordadas y coches arrastrados por las riadas.

$\Rightarrow$ Más de 200 hogares en 4 ciudades afectados por cortes de electricidad.

$\Rightarrow$ Daños materiales importantes. Tres escuelas inundadas cerradas en Vilassar.

Tabla 1: Daños y víctimas (mayoritariamente en el Maresme)

\section{Entorno sinóptico y mesoescalar}

El 12 de octubre de 2016 el contexto sinóptico y mesoescalar del suroeste de Europa estaba regido por el paso de un frente frío asociado a una baja fría que se centraba al noroeste de Portugal (Fig. 3). La baja fría $\left(-20^{\circ} \mathrm{C}\right.$ en $\left.500 \mathrm{hPa}\right)$ fue trasladándose hacia el este/sureste durante el día. Asimismo, en niveles altos (500-300 hPa) durante la mañana del 12 una suave dorsal se situaba sobre el este peninsular y sur de Francia. Dicha dorsal se fue desplazando dejando un área de difluencia sobre Cataluña que favorecía los ascensos. En altura, el flujo predominante era del suroeste. A su vez, en $300 \mathrm{hPa}$ se situaba a las 18 UTC sobre la zona de estudio la parte delantera de un jet streak (máximo de viento confinado).
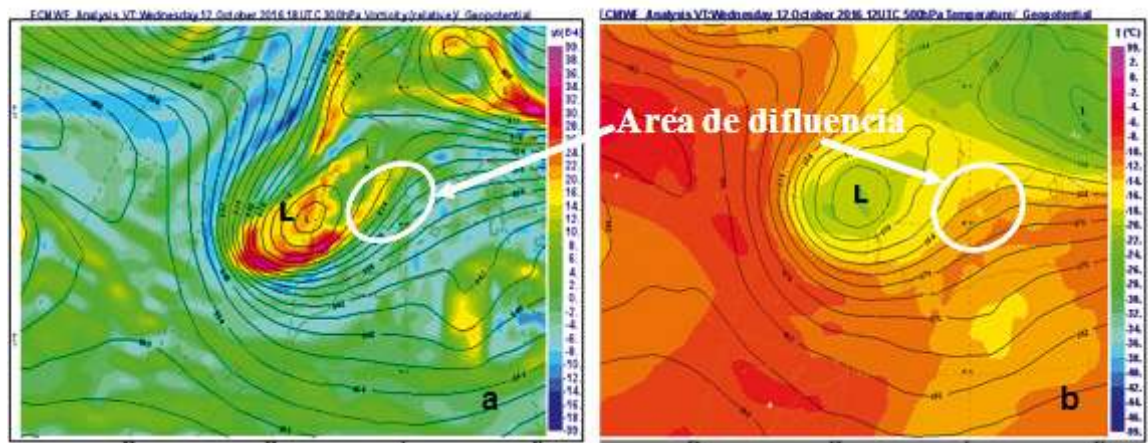

Fig. 3. Análisis del modelo del Centro Europeo IFS-HRES - 12 oct 12 UTC: altura geopotencial y verticidad relativa en $300 \mathrm{hPa}$ (a), y altura geopotencial y $\mathrm{T}$ en $500 \mathrm{hPa}$ (b).

En niveles bajos (Fig. 4), la depresión ligada a la baja fría llevaba asociada un activo frente frío, seguido de dos líneas de inestabilidad post-frontal, que se fue moviendo hacia el este a lo largo del día. Previamente al paso del frente, la confluencia de flujos de recorrido mediterráneo generó un chorro en niveles bajos o Low Level Jet (LLJ) junto con una línea de inestabilidad que fue barriendo el litoral catalán a lo largo de la tarde. Dicha línea y el LLJ contribuyeron a originar los fuertes chubascos, incluso torrenciales, que afectaron al Maresme. Sobre la zona de estudio también había convergencia del flujo de humedad hasta $850 \mathrm{hPa}$. 

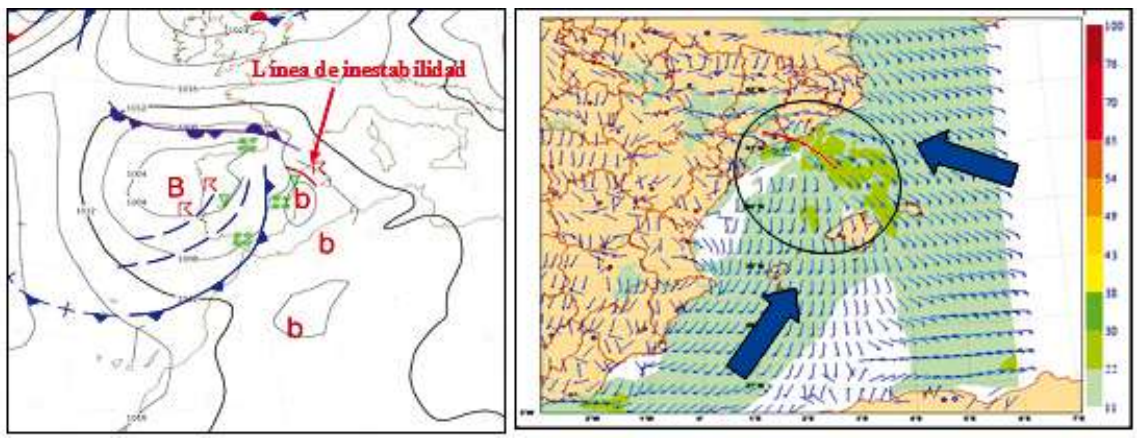

Fig. 4. A la izquierda, análisis frontal de superficie (AEMET) y MSLP (12 oct 12 UTC). A la derecha, campo de viento del modelo Harmonie a las $18 \mathrm{UTC}(\mathrm{H}+18)$

Otros factores mesoescalares que pudieron contribuir a la intensidad y copiosidad de las precipitaciones fueron una temperatura de la superficie del mar (SST) $3^{\circ} \mathrm{C}$ mayor que la media de octubre para la zona y la presencia de la cadena montañosa litoral, con desniveles desde el mar de hasta $700 \mathrm{~m}$ en solamente $7 \mathrm{~km}$ de distancia en línea recta. En condiciones inestables el forzamiento orográfico que sufren los flujos procedentes

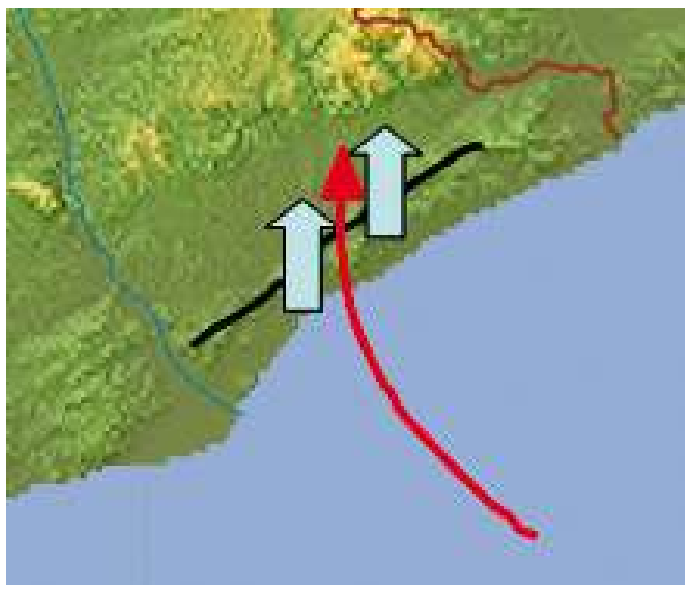
del mar facilita el disparo de la convección (Fig. 5).

Fig. 5. Disparo de la convección debido al forzamiento orográfico de la cadena litoral en la zona de estudio

\section{Fenomenología}

Para este estudio se hizo un análisis de la nubosidad, estructuras precipitantes y rayos en base a las herramientas de teledetección. En el análisis de las imágenes de los canales VIS0.6 e IR10.8 del Meteosat 10 (Fig. 6) se puede apreciar la banda nubosa asociada al frente desplazándose hacia el este. El movimiento de las nubes era hacia el nordeste debido al flujo predominante en niveles medios-altos (del sudoeste). En la imagen del canal visible se aprecia con dificultad el cumulonimbo responsable de las fuertes lluvias en el nordeste de Cataluña, embebido en la parte delantera de la banda nubosa frontal. Las temperaturas de brillo de los topes nubosos de dicho cumulonimbo no muy bajas (estaban comprendidas entre -52 y $-56^{\circ} \mathrm{C}$ ) indican que la convección no era muy profunda. 

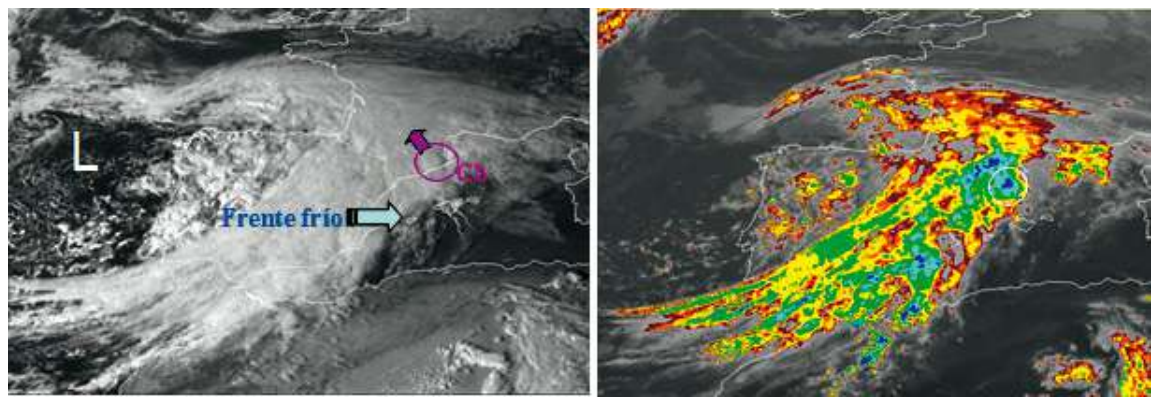

Fig. 6. Imágenes satélite Meteosat10: a la izquierda, imagen del canal VIS0.6 (15:30 UTC); a la derecha, imagen del canal IR10.8 (17:00 UTC).

El análisis de las imágenes del radar de AEMET situado en Cataluña (banda C, rango $240 \mathrm{~km}$ (modo normal)/ $120 \mathrm{~km}$ (modo Doppler)) revela que la acumulación total de precipitación en el Maresme fue subestimada por el radar $(64 \mathrm{~mm}$ estimados frente a los $215 \mathrm{~mm}$ registrados). Esto se debió probablemente a la atenuación del haz radar a causa de la intensa precipitación y a la producida por la película de agua sobre la superficie exterior del radomo. En el análisis de imágenes se puede apreciar una célula convectiva cuasi-estacionaria (Fig. 7) con valores relativamente bajos o moderados de reflectividad, VIL y Echotop durante todo el ciclo de vida de la célula. Aunque el campo de velocidad radial Doppler muestra claramente un desplazamiento hacia el nordeste de la precipitación, los chubascos de mayor intensidad se mantuvieron prácticamente estacionarios debido a la persistencia del LLJ.
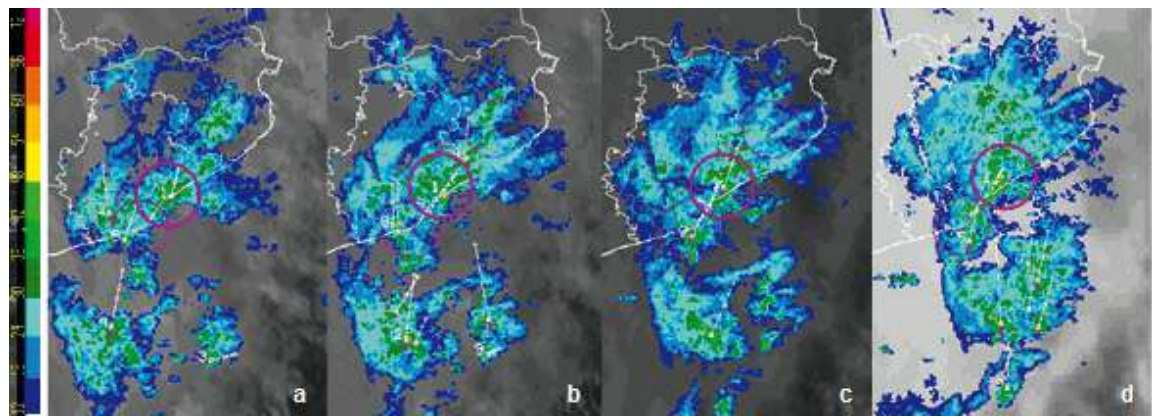

Fig. 7. Producto radar de reflectividad PPIO (dBZ): 15:30 (a), 15:50 (b), 16:10 (c) y 16:30 UTC (d).

Por último, el análisis de rayos nube-tierra caídos durante el episodio (no mostrado) reveló una baja densidad, así como una baja frecuencia de rayos asociados a la tormenta, y un alto porcentaje de rayos positivos. Asimismo se pudo apreciar la cuasi-estacionariedad y aislamiento de la tormenta, pues la más cercana estaba a más de $90 \mathrm{~km}$ mar adentro (hacia el sureste). 


\section{Predicción determinista y probabilista de la precipitación}

En AEMET se usan operacionalmente tanto el modelo determinista del Centro Europeo IFS-HRES (9 km de resolución horizontal) como el modelo mesoescalar no hidrostático HARMONIE-AROME (2,5 km de resolución horizontal; ciclo 38 para este estudio). Se observó que ambos modelos mostraban un área de intensa precipitación moviéndose durante el día 12 hacia el nordeste, siguiendo la línea de costa en Cataluña, pero presentaban ambos un retraso de más de $3 \mathrm{~h}$ respecto al periodo en que se dio la mayor intensidad de precipitación. También ambos subestimaron la intensidad respecto a los valores observados: $40 \mathrm{~mm} / 3 \mathrm{~h}$ el IFS-HRES (Fig. 8), y algo más realista el HARMONIE con $120 \mathrm{~mm} / 3 \mathrm{~h}$ (Fig. 9).
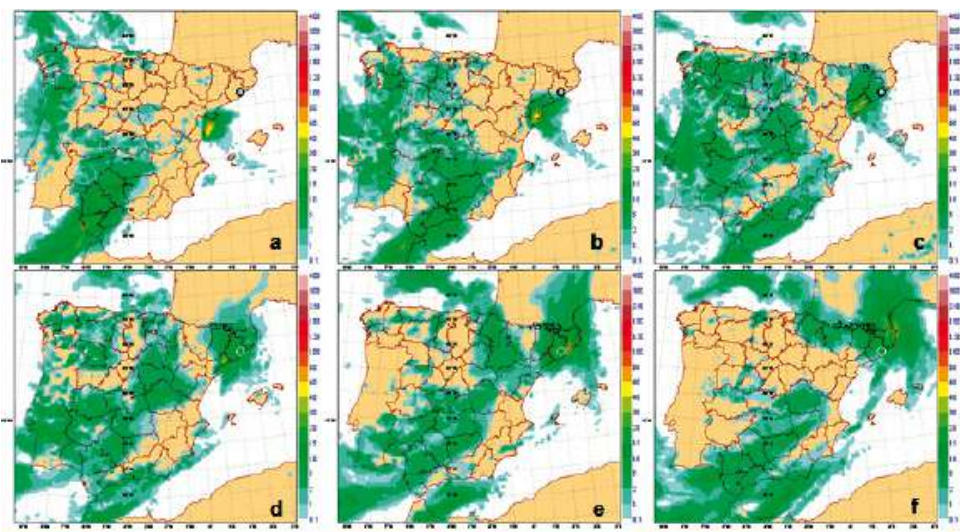

HRES (IFS) ECMW F. 2016101200 UTC. Acumulación de pcp ea 3 h prevista par a 20161012: 6-9 UTC (a), 9-12 (b), 12-15 (c), 15-18 (d), 18-21 (e), 21-24 (f).
Fig. 8. IFS-HRES

(Centro Europeo)

12 oct 00 UTC:

acumulación de pcp en $3 \mathrm{~h}$ prevista para: 6-9

(a), 9-12 (b), 12-15 (c),

15-18 (d), 18-21 (e),

21-24 (f) UTC.

Fig. 9. HARMONIE-

AROME 12 oct 00 UTC: acumulación de pcp en $3 \mathrm{~h}$ prevista para: 6-9 (a), 9-12 (b), 12-15 (c), 15-18 (d), 1821 (e), 21-24 (f) UTC.

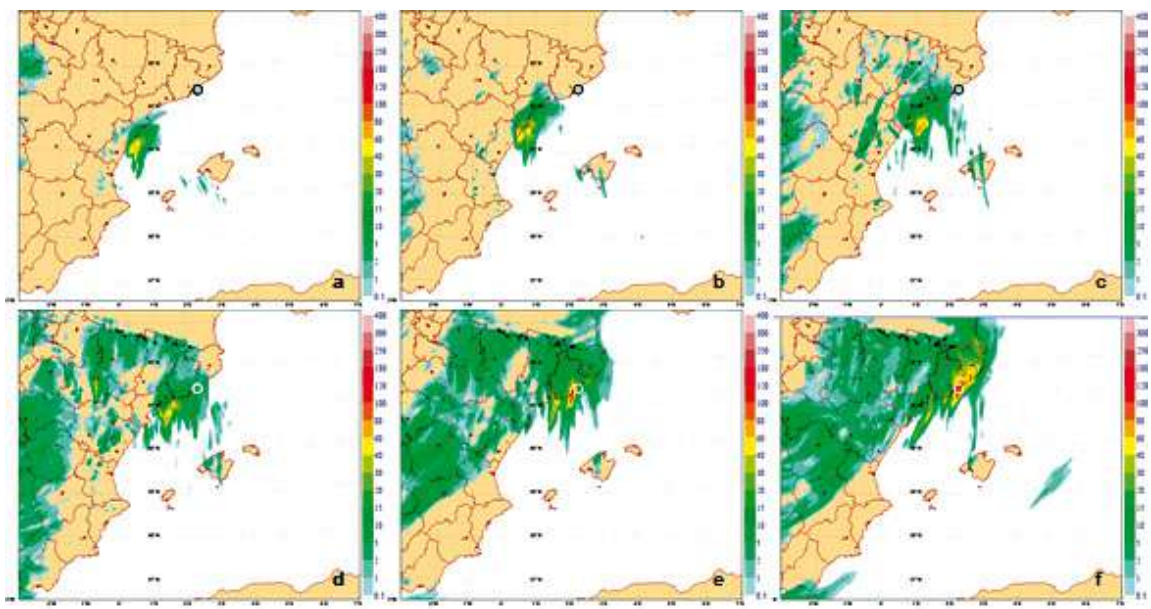


Por otro lado, en este estudio se hizo un análisis desde el punto de vista de la predicción probabilista del sistema ySREPS (desarrollado por Aemet). Se trata de un ensemble de predicción a corto plazo multi-modelo (4 modelos no hidrostáticos) y multi-condiciones de contorno (de 5 modelos globales) compuesto por 20 miembros, posee $2,5 \mathrm{~km}$ de resolución horizontal y tiene la convección explícita. 4 miembros del $\gamma$ SREPS (Fig. 10) mostraron acumulaciones mayores que $50 \mathrm{~mm} / 3 \mathrm{~h}$ y sólo 2 mayores que $120 \mathrm{~mm} / 3 \mathrm{~h}$, todos ellos situando los máximos en un área costera al norte de la ciudad de Barcelona. Cierto número de los miembros presentaba precipitación intensa en áreas costeras y marítimas cercanas.

$\gamma$ SREPS fue integrado para este estudio sin usar ningún método de asimilación, aunque en la actualidad se están implementando el LETKF y el 3-DVAR, lo cual pudo suponer un problema a la hora de simular la precipitación intensa ya que probablemente no tuvo en cuenta algún factor meteorológico mesoescalar local clave en el desarrollo de la convección.
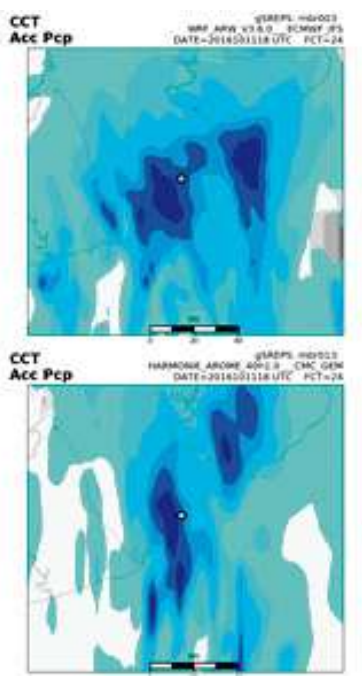

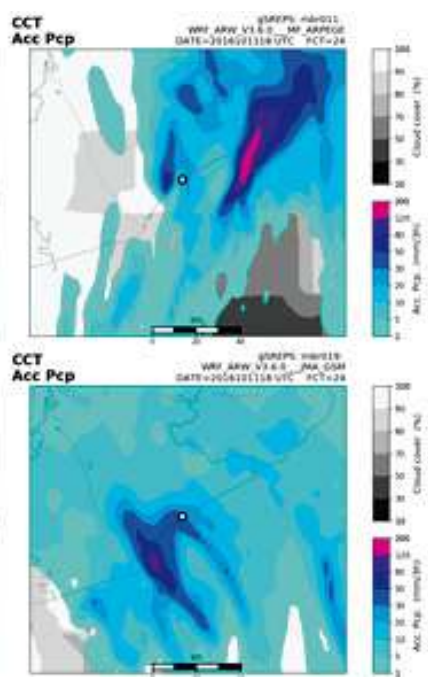

Fig. 10. $\gamma$ SREPS 12 oct 18 UTC. Acumulación en 3 h prevista 15-18 UTC para los 4 miembros con mayor acumulación.

\section{Avisos: un episodio complicado}

El grupo de predicción y vigilancia de AEMET en Barcelona emitió varios avisos de fenómenos meteorológicos adversos a lo largo del episodio. En AEMET existen dos tipos de avisos de precipitación: acumulación de precipitación en $1 \mathrm{~h}$ (P1) y en $12 \mathrm{~h}(\mathrm{P} 2)$, con sus correspondientes umbrales para los distintos niveles de aviso (amarillo, naranja, rojo) para cada zona de aviso.

Ya el día anterior se abrieron para la zona de aviso Litoral de Barcelona (donde se sitúa el Maresme), avisos de nivel naranja de P2 (100 mm/12 h) y de P1 $(40 \mathrm{~mm} / 1 \mathrm{~h})$ 
con una probabilidad superior al $70 \%$. El miércoles 12 a las 16:33 H. L., tras unas horas sin precipitaciones intensas ni tormentas, se decidió bajar el nivel de P1 pero una hora después comenzaron a aparecer indicios de la tormenta en cuestión, lo cual hizo que se elevaran a las 19:19 hora local (H.L.) a nivel rojo los avisos, tanto de P1 (80 mm/1 h) como de P2 $(180 \mathrm{~mm} / 12 \mathrm{~h})$, con una probabilidad del $40 \mathrm{al} 70 \%$. A esa misma hora se estableció que los valores de P1 y P2 a partir de las 20 H.L. se reducían a $20 \mathrm{~mm} / 1 \mathrm{~h}$ y $100 \mathrm{~mm} / 12 \mathrm{~h}$, respectivamente.

\section{Conclusiones}

Este caso de estudio representa un ejemplo de desarrollo de una tormenta con elevada eficiencia en precipitación pese a su modesta profundidad. Se ha observado que dicha tormenta se dio en un entorno sinóptico favorable, pero probablemente el elemento clave en el desarrollo de la precipitación torrencial fue el Low Level Jet (LLJ), que aportó un flujo continuo de aire húmedo hacia la cordillera litoral.

Todos los modelos numéricos usados (tanto deterministas como probabilistas) indicaban la elevada probabilidad de precipitaciones fuertes en la zona de estudio, pero todos subestimaron tanto intensidad como acumulaciones. A su vez, los modelos deterministas presentaron un desfase temporal del máximo de precipitación respecto a lo observado.

Este evento de precipitación torrencial supone un buen ejemplo de la complejidad de algunos episodios meteorológicos a la hora de emitir avisos a la población y a los servicios de Protección Civil. No siempre los modelos numéricos reflejan cuantitativa y/o temporalmente lo finalmente observado, poniendo de manifiesto la importancia de la figura del predictor, tanto en la vigilancia como en el nowcasting.

\section{Referencias}

- Barbería L, Amaro J, Aran M, Llasat MC. 2014. The role of different factors related to social impact of heavy rain events: considerations about the intensity thresholds in densely populated areas. Nat. Hazards Earth Syst. Sci, 14: 1843-1852.

- Chacón S, Gómez S, García S. 2005. Risc d'inundacions als municipis veïns al Parc del Montnegre i el Corredor. Diagnosi ambiental al Parc del Montnegre i el Corredor, 69-75. Diputació de Barcelona: Barcelona.

- Del Moral A., Cortès M., Llasat M.C. y T. Rigo. The 12th October 2016 Maresme flash-floods: a radar-based analysis. Poster presentado en el 10th HyMeX Workshop, 4-7 July 2017, Barcelona, Spain. DOI RG: 10.13140/RG.2.2.14826.82889

- García-Moya, JA, Callado A, Escribà P, Santos C, Santos-Muñoz D, Simarro J. 2011. Predictability of short-range forecasting: a multimodel approach. Tellus A, 63: 550-563. doi:10.1111/j.16000870.2010.00506.x

- Llasat MC, Llasat-Botija M, Rodríguez A, Lindbergh S. 2010. Flash floods in Catalonia: a recurrent situation. Adv. Geosci., 26: 105-111.

- Pascual R. 1999. Estudio de precipitaciones intensas en la comarca catalana del Maresme. Ministerio de Medio Ambiente: Madrid. ISBN: 84-8320-084-8. 